Planta (Berl.) 72, 344-347 (1967)

\title{
DER EINFLUSS DER BODENTROCKENHETT AUF DEN KOHLENHYDRATGEHALT EINIGER CRASSULACEEN
}

\author{
U. KULL \\ Botanisches Institut der Technischen Hochschule Stuttgart
}

Eingegangen am 21. September 1966

\section{THE INFLUENCE OF SOIL MOISTURE ON THE CARBOHYDRATE CONTENT OF SOME CRASSULACEOUS PLANTS}

Summary. Plants of Bryophyllum tubiflorum were held 12 days, plants of Sempervivum tectorum 11 and 23 days without water. Then, the contents of sugars and starch were determined. In Bryophyllum the quantity of monosaccharides decreased, that of oligosaccharides and of starch increased. In Sempervivum the total contents of sugars were higher after Il days, but the amount of starch had decreased. After 23 days of dryness the contents of sugars and of starch had decreased very much, and a somewhat exceptional behaviour of raffinose was observed.

Der Wassergehalt des Bodens hat bekanntlich auf Speicherung und Ablagerungsform der Kohlenhydrate vegetativer Pflanzenteile erheblichen Einfluß (vgl. z.B. LevicT, 1956; IuJıN, 1957). Da über das Verhalten der Sedoheptulose bei Dürrebelastung von Pflanzen bisher außer den Befunden an Coleus (KULL, 1965) keine Angaben vorliegen, schien es von Interesse, die stets Sedoheptulose führenden Crassulaceen in dieser Hinsicht zu untersuchen, nicht zuletzt auch in Hinblick auf deren Sonderstellung als Succulenten. Im Rahmen von Untersuchungen über das physiologische Verhalten der Sedoheptulose bei Crassulaceen (vgl. KuLL, 1966) wurden daher einige orientierende Untersuchungen vorgenommen, über die hier berichtet wird.

$\mathrm{Zu}$ den im Juli durchgeführten Versuchen dienten gleichaltrige kleinere Pflanzen eines Klons von Bryophyllum tubiflorum HaRv. und eingetopfte jüngere Rosetten von Sempervivum tectorum L. Die Ernten des Materials erfolgten stets um 11.00 Uhr. Die papierchromatographische Untersuchung und quantitative Bestimmung der Zucker sowie die Stärkebestimmung erfolgten nach früher beschriebenen Verfahren ( $J_{\mathrm{E}-}$ REMIAS, 1958; JeRemras, 1965; KuLL, 1965). Die Prozentangaben sind auf das Trockengewicht bezogen.

Die Bryophyllum-Pflanzen wurden z. T. normal gegossen, z. T. 12 Tage lang völlig trocken gehalten. Die „Blätter“ (Phyllodien) dieser letzteren Pflanzen waren nicht mehr voll turgescent. Der Gesamtzuckergehalt (vgl. die Abb. 1) zeigt gegenüber demjenigen der Kontrollpflanzen kaum Veränderungen. Jedoch treten deutliche Mengenverschiebungen zwischen den einzelnen Zuckern auf. Der Monosaccharidgehalt nimmt infolge des Verhaltens der Sedoheptulose und noch mehr der Fructose ab, die Menge 
der Oligosaccharide dagegen zu. An dieser Zunahme ist vor allem Raffinose beteiligt, die in den Kontrollpflanzen nur in nicht meßbaren Mengen (ca. 0,05-0,1\%) vorkommt. Die Stärke weist bei den trockengehaltenen Pflanzen eine Zunahme um etwa ${ }^{1 / 5}$ auf.

Beim Versuch mit Sempervivum erfolgten Ernten der trockengehaltenen Pflanzen nach 11 und 23 Tagen. Die Turgescenz der Blätter war im ersten Fall noch nahezu erhalten, nach 23 Tagen dagegen erheblich abgesunken. Die Gesamtmenge der Zucker zeigt bei der ersten Ernte eine

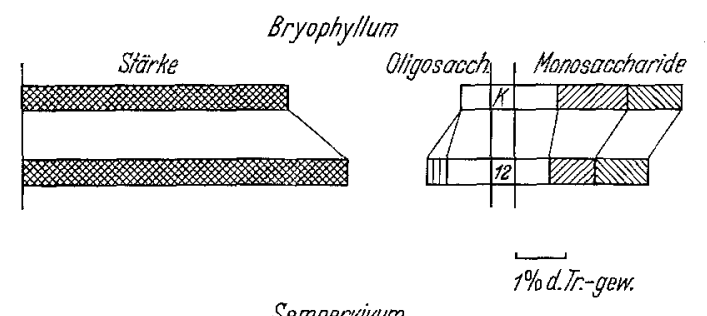

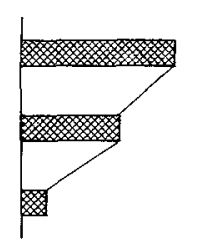

Monosaccharide:

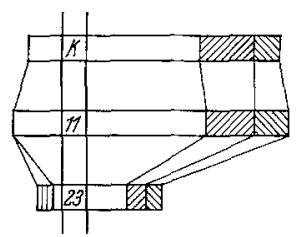

Oligosaccharide;

$$
\begin{aligned}
& \square=\text { Saccharose } \\
& \square] \text { Raffinose }
\end{aligned}
$$

Abb. 1. Verhalten der Kohlenhydrate in Blättern von Bryophyllum tubiflorum und Semperoivum tectorum bei Trockenheit ( $K$ Kontrolle; 11, 12, 23: Tage Versuchsdauer)

geringe Zunahme gegenüber dem Kontrollwert, die vor allem auf das Ansteigen der Glucose- und Saccharosemengen zurückzuführen ist. Der Gehalt an Stärke nimmt um ca. ${ }^{1 / 3}$ (von 2,6 auf 2,0\%) ab. Nach 23 tägiger Dürre ist eine erhebliche weitere Abnahme der Stärke (auf $0,5 \%$ ) sowie eine außerordentliche Verminderung der Zuckergehalte festzustellen. An der letzteren sind alle Zucker beteiligt mit Ausnahme der nur in diesen Pflanzen nachweisbaren Raffinose, die hier in einer Menge von 0,3\% in Erscheinung tritt. Die Abnahme der Speicherkohlenhydrate ist vermutlich auf einen Hungereffekt zurückzuführen, der durch die mehr als dreiwöchige völlige Trockenheit verursacht wurde (vgl. LeviTT, 1956, S. 168).

Die Versuche zeigen, daß wesentliche Veränderungen des Sedoheptulosegehaltes durch Bodentrockenheit bei den untersuchten Arten nicht auftreten, im Gegensatz zu dem bei Coleus blumei beobachteten Verhalten (KuLL, 1965). Coleus reagiert als Hygrophyt wesentlich empfindlicher als die succulenten Crassulaceen und zeigt die vielfach beschriebene 
Zunahme der löslichen Kohlenhydrate (vgl. LeviTT, 1956; ILJIN, 1957) sehr deutlich. Das andersartige Verhalten der beiden Crassulaceen ist ein weiteres Beispiel dafür, daß sich die Bodentrockenheit sehr verschieden auf den Kohlenhydratstoffwechsel der Pflanzen auswirken kann (vgl. HENCKEL, 1964; JEREMIAS, 1966).

Von besonderem Interesse scheint das Auftreten von Raffinose bei den trockengehaltenen Pflanzen. Unter normalen Bedingungen tritt dieser Zucker in Bryophyllum-Blättern nur in Spuren, in Blättern von Sempervivum während der Sommermonate gar nicht auf. Dagegen wird er in den letzteren während der kälteren Jahreszeit gespeichert (KoLL, 1966). Ob das Auftreten bei Dürrebelastung der Pflanzen mit einer Resistenzerhöhung in Zusammenhang gebracht werden kann, muß offen bleiben. Es ist jedoch denkbar, daß den Raffinosezuckern in dieser Hinsicht Bedeutung zukommt (JEREMIAS, 1966), ähnlich einer möglichen Funktion bei der Erhöhung der Frostresistenz (JEREMIAs, 1964). Besonders das Verhalten von Sempervivum weist in diese Richtung. Die Ursache der Anhäufung von Galaktosiden kann darin zu suchen sein, daß infolge der Verhinderung des Wachstums rasch verwertbare Kohlenhydrate, wie es gerade die Raffinosezucker sind, gespeichert werden. Diese Wachstumshemmung ist in unserem Fall bedingt durch Wassermangel, im Fall der winterlichen Speicherung vermutlich durch die Temperaturverhältnisse im Zusammenwirken mit endogenen Faktoren, die zu einer Aktivitätsverringerung der Zellen führen (vgl. BüNNING, 1953). Es ist denkbar, daß die galaktosehaltigen Oligosaccharide spezifische Wirkungen entfalten können, wobei an einen Wirkungsmechanismus entsprechend den Vorstellungen von Heber (1963) und Heber u. Samtarius (1964) zu denken wäre. Auch die winterliche Anhäufung von Galaktolipiden (vgl. KULL, 1965, S. 243) könnte dann in dieser Richtung zu deuten sein. In diesem Zusammenhang scheinen die Befunde von Babenko u. Gevor$\overline{\mathrm{K}} \mathrm{YAN}$ (1966) von Interesse.

Das Verhalten von Bryophyllum im Dürreversuch kann z.T. mit den Untersuchungen von JEREMMAs (1966) an Teucrium verglichen werden, wo eine deutliche Vermehrung von Oligosacchariden, vor allem der Stachyose, zu finden war. In Bryophyllum tritt an deren Stelle die Raffinose. Eine Stärkezunabme bei Wassermangel wurde an anderen Objekten ebenfalls beobachtet; sie könnte darauf zurückzuführen sein, daß durch die Trockenheit zwar eine Wachstumshemmung eintritt, die Photosynthese jedoch zunächst weniger beeinflußt wird (JEREMras, unveröffentlicht; vgl. LEviTT, 1956).

Für verschiedene Anregungen und Diskussionen danke ich Herrn Prof. Dr. A. ARNold und Herrn Priv.-Doz. Dr. K. Jeremias, letzterem auch für die Frlaubnis zur Einsicht in unveröffentlichte Ergebnisse. Für Sachbeihilfen bin ich der Deutschen Forschungsgemeinschaft zu Dank verpflichtet. 


\section{Literatur}

Babenko, V. I., and A. M. Gevorkyar: Carbohydrate metabolism in winter wheat plants in the process of their hardening and de-hardening. Dokl. Vses. Akad. Selskokhoz. Nauk, (1966) 6-11. Zit. nach Chem. Abstr. 65, 1052a (1966).

BünNing, E.: Entwicklungs- und Bewegungsphysiologie der Pflanze. BerlinGöttingen-Heidelberg: Springer 1953.

Heber, U.: Biochemical and physiological aspects of plant frost resistance. Abstr. Intern. Symp. Cytoecology, Leningrad 1963. Moscow: Acad. Sci. USSR Press 1963, p. 27-29.

-, and K. A. Santarius: Loss of adenosine triphosphate synthesis caused by freezing and its relationship to frost hardiness problems. Plant Physiol. 39, $712-719$ (1964).

Henckel, P. A.: The physiology of plants under drought. Ann. Rev. Plant Physiol. 15, 363-386 (1964).

JeREMTAS, K.: Ưber den Jahresgang einiger Zucker in den Blättern von Hedera helix L. Planta (Berl.) 52, 195-205 (1958).

- Über die jahresperiodisch bedingten Veränderungen der Ablagerungsform der Kohlenhydrate in vegetativen Pflanzenteilen (unter besonderer Berücksichtigung der Zucker der Raffinose-Gruppe). Bot. Studien (Jena) 15, 1-96 (1964).

- Der Einfluß des Welkens auf den Zucker- und Stärkegehalt isolierter Blätter von Teucrium chamaedrys und Brunella grandiflora. Planta (Berl.) 65, 73--82 (1965).

- Der Einfluß der Bodentrockenheit auf den Zuckergehalt vegetativer Pflanzenteile. Z. Pflanzenphysiol. 54, 237-239 (1966).

ILJIN, W. S.: Drought resistance in plants and physiological processes. Ann. Rev. Plant Physiol. 8, 257-274 (1957).

KuLx, U.: Über das Vorkommen und das physiologische Verhalten der Sedoheptulose im Rahmen des Kohlenhydrathaushaltes vegetativer Pflanzenteile. Beitr. Biol. Pflanz. 41, 231-300 (1965).

- Zum physiologischen Verhalten der Sedoheptulose im Rahmen des Kohlenhydrathaushaltes einiger Crassulaceen. Ber. dtsch. bot. Ges. 79 (1966) (im Druck).

Levitt, J.: The hardiness of plants. Agronomy VI. New York: Academic Press 1956.

Dr. U. KULL

Botanisches Institut der TH Stuttgart

7 Stuttgart-Ost, Cannstatter Str. 21.2 